\title{
Exosomes: recruits for tumour surveillance?
}

\section{Francesco Pezzella}

Division of Nuffield Clinical Laboratory Science, Department of Radcliffe Medicine, John Radcliffe Hospital, Headington, Oxford, UK

Correspondence to: Francesco Pezzella. Division of Nuffield Clinical Laboratory Science, Department of Radcliffe Medicine, John Radcliffe Hospital, Headington, Oxford OX3 9DU, UK. Email: francesco.pezzella@ndcls.ox.ac.uk.

Provenance: This is an invited Editorial commissioned by Section Editor Dr. Chunlin Ou (Cancer Research Institute of Central South University, Changsha, China).

Comment on: Zhang Z, Li X, Sun W, et al. Loss of exosomal miR-320a from cancer-associated fibroblasts contributes to HCC proliferation and metastasis. Cancer Lett 2017;397:33-42.

Submitted Oct 05, 2017. Accepted for publication Oct 10, 2017.

doi: $10.21037 /$ jtd.2017.10.24

View this article at: http://dx.doi.org/10.21037/jtd.2017.10.24

Extracellular vesicles were firstly described 50 years ago (1). Subsequently it was discovered that three different types could be distinguished: microvesicles and apoptotic bodies (larger than $100 \mathrm{~nm}$ ) and exosomes (up to $150 \mathrm{~nm}$ in diameter) (2). Exosomes are small vesicles derived from, and formed inside, the endosomal compartment (Figure 1A). Endosomal vesicles containing exosomes are called Multivesicular Bodies (MVBs) and they fuse with the cell membrane releasing the exosomes outside the cell (2). Exosomes can subsequently be uptaken by other cells trough clathrin-independent pathways (Figure 1B) (3). In this way their content can be transferred from cell to cell $(2,5)$. Firstly formally described in 1987 (6) they were, according to a pattern very common in science, first dismiss as artefact and subsequently as of no interest (7). It has been only after some years that their importance has been eventually acknowledged $(2,5,7)$.

\section{Communicating by exosomes}

Exosomes represent a very original way in which cells can communicate (8). These vesicles contain a large variety of molecules, not only the like of growth factors, transcription factors or hormones but also nucleic acids, most notably mRNA, miRNA and DNA [mitochondrial, single stranded, transposable elements and large (>10 kb) double strands] (9). Once transferred in a new cell, these factors and nucleic acids, according to their ability, will affect pathways regulation and eventually the cell behaviour. The main difference from the other communication systems is that active molecules from inside the cells are transferred; therefore the cell does not receive a stimulus but the end product. In this way a cell which is, e.g., proliferating because of hormonal stimulation can, trough exosomes, trigger proliferation in a nearby cell which has no receptors for the hormone in question, by transferring, e.g., an activated intracellular protein.

A classic example is that of miRNA, a family of noncoding RNA with an average length of 20-22 nucleotides (10). MiRNAs are directly implicated in tumorigenesis by acting as oncogenic or tumour suppressors (TSGs). Because miRNA function is to inhibits the expression of a protein by silencing mRNA, an oncogenic miRNA (i.e., one those over expression induces cancer) in one that blocks production of a TSG protein. A TSG miRNA (i.e., one those loss lead to cancer) is one blocking the production of an oncogene. Therefore the transfer, or lack of transfer, of a miRNA can have a profound impact on the functions of the receiving cell by altering the expression of genes trough silencing (or failing to silence) the translation of mRNA into protein (10).

Another example of the important role played by exosomes is their ability to transfer epigenetic changes. Epigenetic regulation affects the expression of genes without affecting the nucleotide sequence of the genome, the best known example being methylation of DNA. Hypermethylation of a promoter region inhibits transcription while hypomethylation enhances it. DNA methyltransferase 1 (DNMT1), DNA methyltransferase 3a (DNMT3a), and DNA methyltransferase 3b (DNMT3b) are three among the enzymes able to add methyl groups 


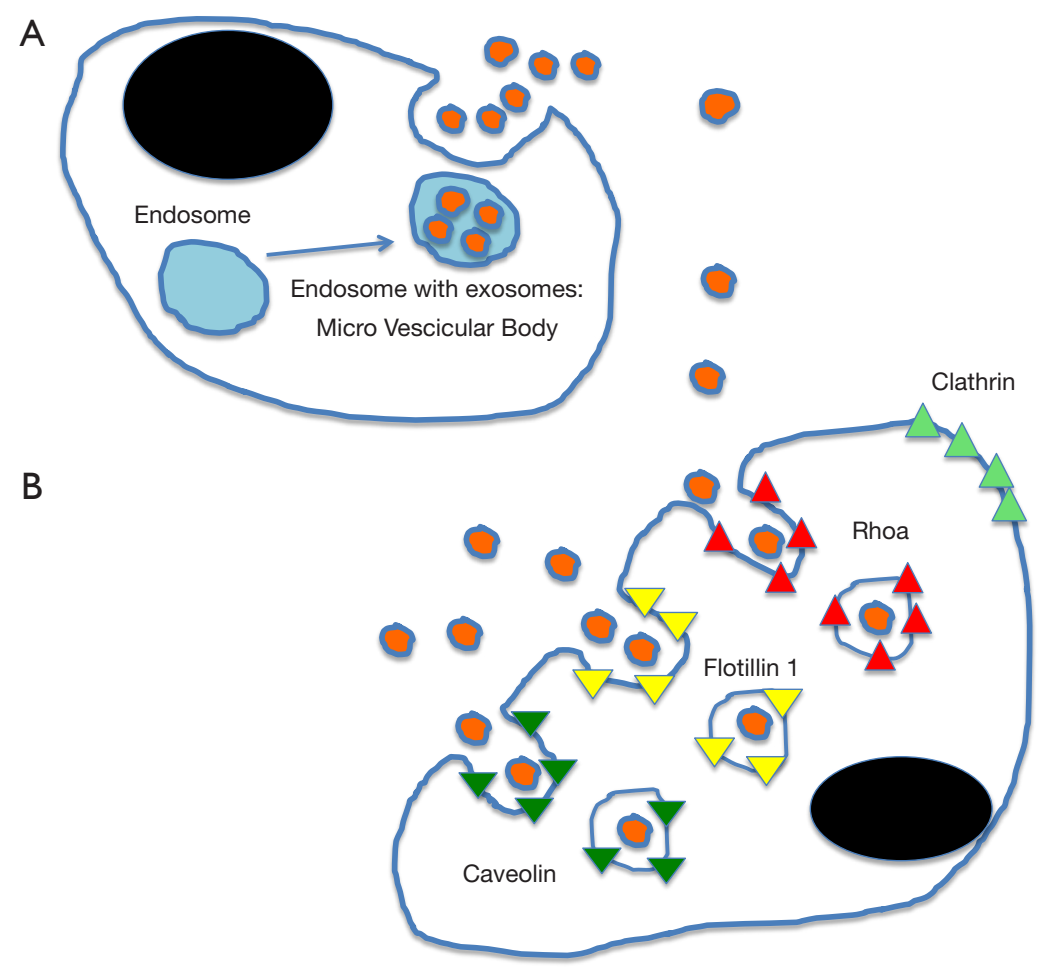

Figure 1 Exosomes formation and uptake. Based on references (2-4). (A) Exosomes (orange) are formed inside the endosomes and are lined by cellular membrane (blue). Endosomes-containing exosomes are called Microvesicular Membrane: as their membrane fuses with the cell membrane the exosomes are released; (B) exosomes are than uptake by a second cell through endocytosis. This process is clathrinindependent and is mediated by other molecules like Caveolin, Fotillin 1 and RhoA.

to specific cytosine in the $\mathrm{CpG}$ islands of regulatory sequences. Exosomes transfer has been demonstrated to be associated with increases in the level of these three enzymes and consequent hypermethylation. As treatment of the exosomes with RNAs reverse the increases of these enzymes and their activity, this effect depend on the transfer of RNA by exosomes (11). Therefore exosomes not only can transfer characters horizontally from on cell to another, but can induce epigenetic changes in the receiving cell, and these changes are known to be transmissible to the progeny (12).

\section{The exchange of characters}

Cells can produce exosomes which get transferred to the nearby cell altering the phenotype of the receiving cell, i.e., transferring a character acquired by the donating to the recipient cell (13): this is a very interesting fact. The French naturalist Jean-Baptist Lamarck [1744-1829] thought, as Darwin after him, that species changes with time and new species derivate from old ones although, contrary to
Darwin, he thought that each specie transform in a new one and so on, without extinction or branching. The main mechanism for Lamarck was an unknown "internal force" while a second mechanism, less important to him, was the possibility to transmit acquired characters (14). The transmission of acquired characters has been regarded as impossible for long time but now it has been demonstrated that acquired epigenetic changes, like DNA methylation, can be transmitted from a cell to its progeny (12). The recent work on exosomes suggests that such a type of acquired character can be also transmitted horizontally. This is not only important for the understanding of how tumours form but also could be of extreme interest in the field of treatment resistance as theoretically an acquired epigenetic change due to exposure to a drug could be passed on to a cell never exposed to such a drug.

\section{Exosomes and the TSG miRNA-230a}

In a paper recently published Zhang et al. (15) investigated 
the role of exosomes as messenger between fibroblasts and neoplastic cells as far as the transfer of miR-320a is concerned. Stroma plays an important role in cancer and differences in miRNA profile between those fibroblasts located inside the tumour and the one in the normal tissue have been found $(16,17)$. Such a difference is now confirmed also in the exosomes derived from the cancer-associated stromal fibroblasts (CAFs), harvested from the neoplastic lesion, and the matched para-cancer normal fibroblasts (NAFs), defined as fibroblasts from adjacent tumour-free tissue, at least $5 \mathrm{~cm}$ from the edge of the tumour. Among the miRNA differentially expressed the authors found the miR-320a. Their data show that it is down regulated in the CAFs derived exosomes compared to those released from the nearby normal tissue fibroblasts. Therefore the authors demonstrated that the exosomes received by the cancer cells from the CAFs, have lower levels of miRNA-320a compared to the exosomes released from normal-tissue fibroblasts, located at greater distance from the lesion.

This miRNA has an inhibitory effect on proliferation and motility and is involved in the induction of apoptosis (18). It has been found to be down regulated in breast, colorectal tumours, hepatocellular carcinomas (13) and multiple myeloma (18). So far two targets genes have been identified: $\mathrm{cMyc}$, whose role as oncogene needs no introduction and PBX3 (19) which belong to the Pre-B-cell leukemia homeobox family PBX. PBX3 is downstream to, and is activated by, the ERK1/ERK2 complex in the MAPK pathway (19).

CAFs were for many years considered as bystander but recently it has emerged that they have an active role in cancer and there is cross talking between CAFs and the neoplastic cells. CAFs are activated cells and show an increased proliferation and motility, they have been reported to have both a tumour-promoting and a tumourinhibiting effects, according to the setting (20). Some of the way in which CAF contribute to cancer growth are oncogenic-like, i.e., dominant, like increased secretion of metalloproteases, favouring invasion (21) or of growth factors inducing proliferation (22). These changes mostly reflect the activated nature of the CAFs.

Zhang et al. have reported that miRNA-230a is down regulated in the CAFs compared to the NAFs. In view of the fact that miRNA-230a contributes to suppress cell proliferation and motility, it is not surprising that activated CAF would, as part of the changes linked to their activated status, down regulate this miRNA. As the level of miRNA in the exosomes reflects that of the cell releasing it, this means that CAF released exosomes will have a diminished tumoursuppressor effect compared to that released by NAFs (15). This implies that NAF could exert a physiological "tumour suppressor" activity to maintain homeostasis in the tissue (15) and that the tumour-promoting effect reported by Zhang and co-workers when loss of this miRNA occurs, is "visible" because a suppressor activity is lost.

\section{Cancer surveillance by exosomes?}

This brings to the issue of whether NAF exerts physiologically such a TSG activity and how. Experimental model have been reported in which NAFs have an inhibitory effect on cancer growth.

In a 3D "in vitro" model Sadlonova et al. co-cultured the MCF10A non-tumorigenic human breast epithelial cell line and the human MCF10AT malignant breast epithelial line with either CAF or NAF, derived from normal (nonneoplastic) mammary tissue. Both NAFs and CAFs showed inhibitory property on the growth but to different degrees. Both NAFs and CAFS could inhibit the growth of the low grade MCF10A cells but only the NAFs could block the growth of the high grade neoplastic cells MCF10AT. The authors speculate that NAFs are more effective in tumour suppression and this suppressive ability may be lost or reduced as epithelial lesions gradually progress from hyperplasia to invasive cancer, and accordingly NAF are activated to transform into CAFs (23). In a second study using the same two cell lines but this time grafted in a mouse, Sadlonova et al. implanted the tumours with NAFs and compared their effect on low and high grade lesions. The low grade MCF10A xenografts, when infiltrated with NAFs, grew less and showed an increased number of apoptotic cells but this effect was much reduced in the higher grade lesions derived from the MCF10AT cells (24). The author speculate that this could be due to the more aggressive phenotype of the MC10AT cells, becoming increasingly resistant to the NAFs effect on one side, and on the other side to the ability of the high grade cancer cells to progressively induce the NAFs to acquire a CAF phenotype. Across the literature, these effects can change according to the cell lines used plus there is variation from patient to patient, on the ability of their NAFs to inhibit growth $(23,24)$.

Working on a prostate cancer models, broadly comparable results have been found (25). Using "in vitro" cultures, low grade growth of immortalised epithelial cells or cells from benign prostatic hyperplasia, could be 

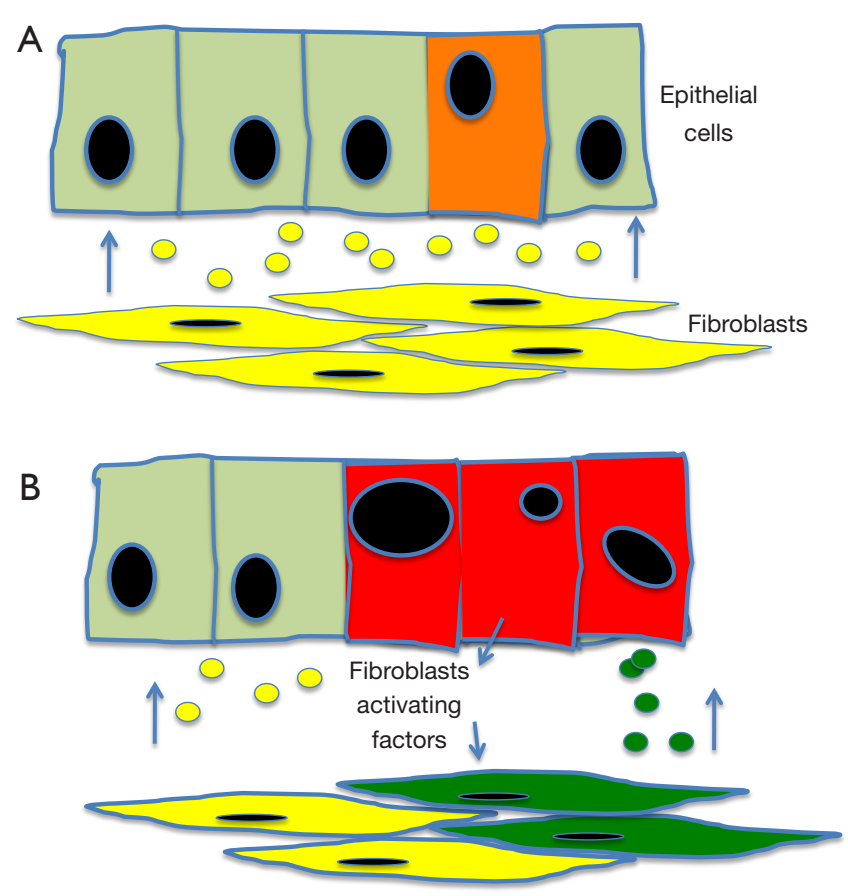

Figure 2 A hypothetical mechanism by which fibroblasts could participate in cancer surveillance. Based on references $(15,23)$. (A) PAF (normal tissue fibroblasts, yellow) secretes exosomes (in yellow) containing tumour suppressor factors like miRNA-320a. An early transformed cell is present (orange) but is still unable to induce fibroblasts stimulation. The uptake of exosomes containing suppressor factors could contributes to maintain the cell quiescent or could even induce apoptosis; (B) transformed cells, which have acquired further damages and a more neoplastic phenotypes (in red) are now able to secreted fibroblasts activating factors. The activated fibroblasts (green) secrete now exosomes with lower levels of miRNA320a (also in green) and therefore their suppressive ability is diminished, giving to the neoplastic cells further advantage.

inhibited by NAFs but not by CAFs. When comparing the effect of NAFs on the low grade and on the high grade metastatic prostate cancer cells PC-3, NAFs could again block the growth of the low grade but failed to inhibit the high grade cells. CAFs failed again to inhibit the low grade cells from growing. At variance with the breast model above discussed, CAFs could instead inhibit the expansion of the high grade metastatic PC-3 cell line. The supernatant from the cultures was found to have the same property of the fibroblasts growing into it, indicating the presence of a soluble factor(s) (25).

These studies point to the ability of NAFs to inhibit early transformed cells. The paper from Zhang and co-workers raises the hypothesis that exosomes containing miRNA320 a could be one of the mechanisms by which NAFs may help containing neoplastic transformation. According to this hypothesis the secretion by exosomes of TSG mediators, in this case miRNA-320a, could help to either eliminate, e.g., by inducing apoptosis, or maintain quiescent, e.g., by inhibiting cell cycle, early transformed cells. However, if further cellular damage occurs, the transformed cells could, at same stage, influence the normal cell and turn down the suppressor stimulus. In the case of the interaction with fibroblasts, the tumour cells could acquire a change, which release messenger stimulating the quiescent fibroblasts and pushing them into proliferation and migration. As a consequence, suppressor factors like miRNA-320a are down regulated and their suppressor activity is lost, providing further advantage to the transformed cell (Figure 2).

Our understanding of the role of exosomes, and indeed of their biology, is at the very beginning. The paper from Zhang and coworker open a new exciting area in this field by suggesting for the exosomes a role in tumour surveillance.

\section{Acknowledgements}

None.

\section{Footnote}

Conflicts of Interest: The author has no conflict of interest to declare.

\section{References}

1. Wolf $\mathrm{P}$. The nature and significance of platelet products in human plasma. Br J Haematol 1967;13:269-88.

2. Hessvik NP, Llorente A. Current knowledge on exosome biogenesis and release. Cell Mol Life Sci 2017. [Epub ahead of print].

3. Costa Verdera H, Gitz-Francois JJ, Schiffelers RM, et al. Cellular uptake of extracellular vesicles is mediated by clathrin-independent endocytosis and macropinocytosis. J Control Release 2017;266:100-8.

4. Smythies J, Edelstein L, Ramachandran V. Molecular mechanisms for the inheritance of acquired characteristicsexosomes, microRNA shuttling, fear and stress: Lamarck resurrected? Front Genet 2014;5:133.

5. Mayor S, Parton RG, Donaldson JG. Clathrinindependent pathways of endocytosis. Cold Spring Harb 
Perspect Biol 2014;6:a016758.

6. Johnstone RM, Adam M, Hammond JR, et al. Vesicle formation during reticulocyte maturation. Association of plasma membrane activities with released vesicles (exosomes). J Biol Chem 1987;262:9412-20.

7. Johnstone RM. Revisiting the road to the discovery of exosomes. Blood Cells Mol Dis 2005;34:214-9.

8. Sullivan R, Maresh G, Zhang X, et al. The Emerging Roles of Extracellular Vesicles As Communication Vehicles within the Tumor Microenvironment and Beyond. Front Endocrinol (Lausanne) 2017;8:194.

9. Kahlert C, Melo SA, Protopopov A, et al. Identification of double-stranded genomic DNA spanning all chromosomes with mutated KRAS and p53 DNA in the serum exosomes of patients with pancreatic cancer. J Biol Chem 2014;289:3869-75.

10. Takahashi RU, Prieto-Vila M, Hironaka A, et al. The role of extracellular vesicle microRNAs in cancer biology. Clin Chem Lab Med 2017;55:648-56.

11. Qian Z, Shen Q, Yang X, et al. The Role of Extracellular Vesicles: An Epigenetic View of the Cancer Microenvironment. Biomed Res Int 2015;2015:649161.

12. Gapp K, Bohacek J. Epigenetic germline inheritance in mammals: looking to the past to understand the future. Genes Brain Behav 2017. [Epub ahead of print].

13. Xie F, Yuan Y, Xie L, et al. miRNA-320a inhibits tumor proliferation and invasion by targeting c-Myc in human hepatocellular carcinoma. Onco Targets Ther 2017;10:885-94.

14. Ridley M. Evolution. Malden, MA: Balckwell Scientific Publishing; 2004.

15. Zhang Z, Li X, Sun W, et al. Loss of exosomal miR-320a from cancer-associated fibroblasts contributes to HCC proliferation and metastasis. Cancer Lett 2017;397:33-42.

16. Zhao L, Sun Y, Hou Y, et al. MiRNA expression analysis

Cite this article as: Pezzella F. Exosomes: recruits for tumour surveillance? J Thorac Dis 2017;9(11):4295-4299. doi: $10.21037 /$ jtd.2017.10.24 of cancer-associated fibroblasts and normal fibroblasts in breast cancer. Int J Biochem Cell Biol 2012;44:2051-9.

17. Chou J, Werb Z. MicroRNAs play a big role in regulating ovarian cancer-associated fibroblasts and the tumor microenvironment. Cancer Discov 2012;2:1078-80.

18. Lu Y, Wu D, Wang J, et al. miR-320a regulates cell proliferation and apoptosis in multiple myeloma by targeting pre-B-cell leukemia transcription factor 3 . Biochem Biophys Res Commun 2016;473:1315-20.

19. Han $\mathrm{HB}, \mathrm{Gu}$ J, Ji DB, et al. PBX3 promotes migration and invasion of colorectal cancer cells via activation of MAPK/ERK signaling pathway. World J Gastroenterol 2014;20:18260-70.

20. Mesker WF, Tollenaar RAEM. Cancer Associated Stroma. In: Press OU, editor. Oxford Textbook of cancer biology. Oxford: Oxford University Press; In press.

21. Marsh T, Pietras K, McAllister SS. Fibroblasts as architects of cancer pathogenesis. Biochim Biophys Acta 2013;1832:1070-8.

22. Bhowmick NA, Chytil A, Plieth D, et al. TGF-beta signaling in fibroblasts modulates the oncogenic potential of adjacent epithelia. Science 2004;303:848-51.

23. Sadlonova A, Novak Z, Johnson MR, et al. Breast fibroblasts modulate epithelial cell proliferation in threedimensional in vitro co-culture. Breast Cancer Res 2005;7:R46-59.

24. Sadlonova A, Mukherjee S, Bowe DB, et al. Human breast fibroblasts inhibit growth of the MCF10AT xenograft model of proliferative breast disease. Am J Pathol 2007;170:1064-76.

25. Paland N, Kamer I, Kogan-Sakin I, et al. Differential influence of normal and cancer-associated fibroblasts on the growth of human epithelial cells in an in vitro cocultivation model of prostate cancer. Mol Cancer Res 2009;7:1212-23. 